\title{
Evaluación de conocimientos previos de estudiantes universitarios sobre electrocinética e implicaciones para la enseñanza y el aprendizaje de modelos científicos
}

\author{
Alfonso Pontes Pedrajas \\ Dpto. Física Aplicada, Universidad de Córdoba (apontes@uco.es)
}

\begin{abstract}
This work is part of a broader research aimed at improving the quality of physics learning in the training of first-year engineering students. In the first phase of the project, previous knowledge and reasoning models used by students were investigated, when addressing electrokinetics issues and making predictions about the functioning of various electrical circuits.This has been done using an open-ended questionnaire, which students have completed through the Moodle platform, before receiving formal instruction on the subject. The results obtained in this study indicate that students use spontaneous reasoning and express alternative conceptions that show a lack of understanding of the scientific model of electric current.Therefore, in the next phase of the project, it is considered necessary to apply new educational proposals that favor the progression of the mental models of students and help them to overcome the learning difficulties identified in this work.
\end{abstract}

Keywords: Learning physics, scientific models, electrical circuits, alternative conceptions, students' reasoning.

\section{Resumen}

Este trabajo forma parte de una investigación más amplia orientada a mejorar la calidad del aprendizaje de la Física en la formación de estudiantes de primer curso de ingeniería. En la primera fase del proyecto se han investigado los conocimientos previos y los modelos de razonamiento que utilizan los estudiantes al abordar problemas del tema de electrocinética y hacer predicciones sobre el funcionamiento de varios circuitos eléctricos. Para ello se ha utilizado un cuestionario de preguntas abiertas, que los estudiantes han cumplimentado a través de la plataforma Moodle, antes de recibir instrucción formal sobre el tema. Los resultados obtenidos en este estudio indican que los alumnos utilizan razonamientos espontáneos y expresan concepciones alternativas que ponen de manifiesto la falta de comprensión del modelo cientifico de corriente eléctrica. Por ello, en la siguiente etapa del proyecto, se estima necesario aplicar nuevas propuestas educativas que favorezcan la progresión de los modelos mentales de los estudiantes y les ayuden a a superar las dificultades de aprendizaje detectadas en este trabajo.

Palabras clave: Aprendizaje de la Física, modelos científicos, circuitos eléctricos, concepciones alternativas, razonamientos de los estudiantes.

\section{Fundamento y finalidad}

A lo largo de las últimas décadas la problemática de la enseñanza y el aprendizaje de la electricidad ha sido objeto de frecuentes investigaciones didácticas, porque se ha apreciado la persistencia de numerosas dificultades de aprendizaje, en numerosos países y en diferentes niveles educativos, que se han identificado con diversas denominaciones: concepciones o ideas (previas) no científicas, errores conceptuales, esquemas alternativos, razonamientos superficiales, teorías implícitas, argumentos 
espontáneos y modelos mentales acientíficos, entre otros términos (Duit, Jung y Rhoneck, 1985; Clement, 2000; Treagust, Chittleborough y Mamiala, 2002). Este hecho implica un aprendizaje deficiente de los principales conceptos, principios y modelos científicos que se utilizan para interpretar los fenómenos electromagnéticos (Cudmani y Fontevilla, 1990; López-Donoso y Silva, 2015) y adquiere una especial relevancia en el tema de circuitos eléctricos (Meitoui, Brassard, Levasseur y Lavoie, 1996; Pontes y Pro, 2001; Gunstone, Mulhall y McKittrick, 2009), ya que este tema forma parte del currículo de ciencia y tecnología en educación secundaria y es un tema importante de física general en cualquier carrera universitaria de carácter científico-técnico.

La problemática de las concepciones y modelos mentales de los alumnos sobre la ciencia y su influencia como obstáculos para el aprendizaje significativo de la física ha sido analizada en numerosos trabajos (Hierrezuelo y Montero, 1990; Mei-Hung y Jing-Wen, 2005; Balta, 2015), en los que se pone de manifiesto la necesidad de introducir modificaciones sustantivas en el proceso de enseñanza, tratando de tener en cuenta las ideas y modelos mentales de los estudiantes durante el proceso de aprendizaje y ayudarles a transformar tales ideas en modelos científicos (Zeynep e Ibilge, 2011; Oliva, 2019).

Nuestra preocupación por mejorar la calidad de la educación científica universitaria nos ha llevado, desde hace tiempo, a desarrollar materiales didácticos tales como documentos de trabajo en el aula, guiones de prácticas de laboratorio, materiales de ayuda al estudio y programas de actividades para usar diversos tipos de software educativo, tratando de favorecer el cambio conceptual, la progresión de los modelos de pensamiento del alumnado y el aprendizaje significativo de la física en los estudios universitarios (Pontes, 2014; Pontes, 2017; López-Quintero, Varo y Pontes, 2018). En este contexto, estamos llevando a cabo una investigación educativa, en varias etapas, destinada a implementar estrategias y recursos que permitan mejorar el proceso formativo de nuestros alumnos sobre esta temática, partiendo del análisis de sus conocimientos previos y de las exploración de las principales deficiencias de aprendizaje que muestran los estudiantes antes de estudiar el tema de electrocinética en primer curso de Ingeniería.

El proyecto de investigación del que forma parte este estudio, está orientado a fomentar el aprendizaje significativo de la Física y a desarrollar competencias transversales relacionadas con el uso interactivo de las TIC en las aulas universitarias (Pontes, 2020), tratando de integrar diversos recursos informáticos (como Moodle, CmapTools, Simulaciones Phet y Turnning Point) en la formación de los estudiantes de de ingeniería. En este trabajo sólo se ha utilizado la plataforma Moodle de enseñanza virtual para pasar el cuestionario de conocimientos previos sobre electrocinética antes de abordar este tema en clase. En el mismo curso también hemos utilizado la aplicación CmapTools (Pontes, 2014) para que los alumnos desarrollen mapas conceptuales digitales sobre el tema, tratando de fomentar el aprendizaje reflexivo del modelo de corriente eléctrica y sus aplicaciones en ingeniería, aunque los resultados de esa experiencia se mostrarán en un trabajo posterior específico sobre ese tema.

El objetivo principal de este estudio, que forma parte de la primera etapa del proyecto de investigación, es analizar cuáles son los conocimientos previos, los modelos de pensamiento alternativo y las dificultades de aprendizaje de conceptos básicos de electricidad, que muestran los estudiantes en diversos tipos de tareas como la interpretación de fenómenos, la predicción de hechos, o la resolución de problemas de carácter cualitativo en el tema de circuitos de corriente eléctrica.

\section{Metodología de investigación}

Este estudio se ha desarrollado en el bloque de contenidos de Electricidad de la asignatura Fundamentos Físicos de la Ingeniería II (FFI2), impartida en el $2^{\circ}$ cuatrimestre del primer curso del Grado de Ingeniería Eléctrica, en la Escuela Politécnica Superior de la Universidad de Córdoba (EPSC). Para desarrollar el 
objetivo propuesto anteriormente se ha diseñado una prueba de evaluación del conocimiento inicial de nuestros alumnos sobre electrocinética, destinada al análisis de situaciones problemáticas sobre diferentes tipos de circuitos eléctricos, en torno a los cuales los estudiantes debían responder a diversas cuestiones de carácter abierto, exponiendo sus ideas y razonamientos sobre cada asunto.

Para este estudio se han recogido datos de una muestra global formada por dos grupos de clase, que incluye a un conjunto total de 81 estudiantes ( 15 chicas y 66 chicos), con una edad media de 19,6 años, utilizando un cuestionario formado por 5 problemas cualitativos relacionados con el funcionamiento de diversos circuitos eléctricos. En cada problema se han formulado varias cuestiones, que requieren explicaciones y razonamientos de carácter cualitativo o cuantitativo, de modo que el cuestionario completo está formado por 16 ítems, que permiten explorar las ideas de los estudiantes sobre una amplia gama de tópicos de electrocinética. Los enunciados de tales problemas y los dibujos de los montajes correspondientes se muestran posteriormente junto a las tablas de resultados de cada cuestión. Este cuestionario, cuyos ítems se han seleccionado a partir de un conjunto de actividades de inicio, desarrollo y profundización en el aprendizaje de modelos científicos en dominio de la electricidad (Pontes et al., 2019), se ha pasado al inicio del tema de circuitos eléctricos de corriente continua, antes de recibir instrucción universitaria sobre el mismo, porque se trataba de evaluar el conocimiento previo adquirido por los alumnos en la educación secundaria.

Para pasar el cuestionario se ha utilizado la plataforma Moodle, y los dos grupos de estudiantes han dispuesto de una hora para realizarlo, aunque la mayoría de los participantes han utilizado menos tiempo. La herramienta Moodle es importante para mejorar la calidad de la enseñanza universitaria y desempeña un papel relevante en este proyecto de investigación (Pontes, 2020), ya que permite hacer una combinación adecuada del trabajo presencial desarrollado en aula y del trabajo realizado on-line mediante diversas aplicaciones disponibles en Internet, en donde el alumno puede controlar algunos factores como el lugar, momento y espacio de trabajo (Alducin \& Vázquez, 2014). En efecto esta herramienta TIC pone a disposición del alumnado todos los materiales didácticos que se pueden utilizar en clase o en casa (apuntes, presentaciones, vídeos y programas de actividades,...) y fomenta la comunicación entre profesores y alumnos a través de foros, avisos de novedades y mensajes de todo tipo. Por otra parte la plataforma Moodle permite diseñar conjuntos de cuestiones de todo tipo que se pueden utilizar para elaborar pruebas de evaluación (inical, intermedia o final) y facilita al profesorado la tarea de corregir tales pruebas, ya que las respuestas de los alumnos a cada cuestión quedan registradas en una base de datos. Por ello la hemos usado en este estudio para llevar a cabo la evaluación de los conocimientos previos de nuestros alumnos en el tema de electrocinética.

Para el análisis de datos recogidos con el citado cuestionario se clasificaron las respuestas de los alumnos en cuatro categorías generales: (I) respuestas en blanco y respuestas que incluyen ideas confusas e incoherentes, (II) explicaciones erróneas desde el punto de vista científico o modelos de pensamiento alternativo, (III) respuestas aceptables desde el punto de vista científico pero defícientes en su explicación o argumentación y (IV) respuestas correctas que incluyen una explicación razonable y acorde con el modelo científico de corriente eléctrica. En este proceso de análisis cualitativo y categorización de respuestas han participado tres docentes de la asignatura, con bastantes años de experiencia docente, que han colaborado en el desarrollo de un proyecto de innovación de materiales didácticos para la enseñanza de la electricidad en ingeniería (Pontes et al., 2019). Tras la clasificación de respuestas a cada pregunta del cuestionario, los datos cuantitativos se han analizado con el programa SPSS y se ha realizado un análisis de frecuencias relativas (\%), que permite identificar la extensión de los diferentes modelos de pensamiento en cada ítem. 


\section{Resultados}

A continuación se muestran, en varias tablas, los resultados del análisis de las respuestas de los alumnos a las diversas preguntas que integran los cinco problemas del cuestionario. En la tabla de datos de cada problema se presenta primero el enunciado y después los resultados derivados del análisis de frecuencias correspondientes a las cuatro categorías de respuestas (I, II, III y IV) consideradas para cada item.

\subsection{Análisis de un circuito de varios elementos en serie}

En la la Tabla 1 se muestra el enunciado y el montaje del primer problema (P1), que está orientado a analizar los modelos de pensamiento de los estudiantes sobre el funcionamiento de un circuito de varios elementos conectados en serie, formado por un generador ideal, dos lámparas iguales y una resistencia $\mathrm{R}$ fija o variable. En torno a este montaje, los estudiantes deben hacer predicciones sobre el brillo de las bombillas L1 y L2 situadas a un lado y otro de la resistencia, los cambios en el brillo de tales lámparas al aumentar el valor de la resistencia intermedia y la aplicación de la ley de Ohm para valores extremos de la resistencia.

Tabla 1. Resultados del análisis de un circuito de varios elementos en serie

1. El circuito de la figura está formado por un generador ideal G (que proporciona una tensión constante $\mathrm{V}_{\mathrm{o}}$ ) y dos lámparas iguales L1 y L2 (que tienen una resistencia interna de valor $\mathrm{R}_{\mathrm{L}}$ ) en serie con una resistencia externa variable, cuyo valor inicial es igual que la resistencia de las lámparas $\left(R v=R_{L}\right)$

1.1. Explicar cuál de tales lámparas brilla más al principio.

1.2. Predecir si se produce algún cambio en el brillo de ambas lámparas cuando el valor de la resistencia externa disminuye hasta cero (cable sin resistencia).

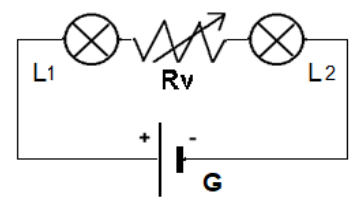

1.3. Predecir qué ocurre en el circuito cuando el valor de la resistencia externa aumenta hasta un valor infinito (equivale a interruptor abierto).

\begin{tabular}{|c|c|c|c|c|c|c|c|c|}
\hline \multicolumn{9}{|c|}{ Frecuencias y Porcentajes por Categorías de Respuesta } \\
\hline Ítems & & & & & & & & \\
\hline 1.1 & 6 & 7,4 & 28 & 34,5 & 22 & 27,2 & 25 & 30,9 \\
\hline 1.2 & 11 & 13,5 & 30 & 37,7 & 24 & 29,6 & 17 & 20,9 \\
\hline 1.3 & 9 & 11,1 & 33 & 40,7 & 20 & 24,7 & 19 & 23,5 \\
\hline
\end{tabular}

En la zona inferior de la Tabla 1 se recogen los resultados derivados de la categorización de ideas en cada uno de estos ítems, con arreglo a los cuatro modelos de respuesta establecidos anteriormente, mostrando las frecuencias y porcentajes de cada categoría que se comentan a continuación.

Con relación a cuál de las dos lámparas brilla más en dicho montaje (ítem 1.1) se aprecia un porcentaje importante de modelos de pensamiento alternativo (Tipo II / MA) o respuestas erróneas desde el punto de vista científico (más de un tercio) y algunas respuestas en blanco o explicaciones confusas (aproximadamente una doceava parte). Respecto a los cambios que se producen en el brillo de las lámparas al aumentar el valor de la resistencia intermedia (item 1.2) los resultados son aun más bajos y algo similar ocurre en la pregunta sobre los cambios que se producen cuando la resistencia intermedia alcanza valores extremos hacia cero o hacia infinito (item 1.3), ya que el porcentaje de concepciones alternativas (Tipo II) es algo superior a dos quintas partes de la muestra.

Los principales modelos de carácter alternativo que se aprecian en estas preguntas se refieren especialmente al modelo de consumo de la corriente eléctrica, ya que muchos estudiantes consideran que "la corriente se va gastando" a medida que va pasando por los diferentes elementos del circuito. En 
segundo lugar las ideas alternativas se relacionan con el razonamiento de tipo local y secuencial, que supone ignorar que los circuitos eléctricos son sistemas físicos interconectados por campos eléctricos, de modo que todas las magnitudes físicas experimentan cambios instantatáneos al modificar cualquier variable independiente (p.e. el valor de la resistencia intermedia). Y en tercer lugar, se puede deber a la falta de comprensión significativa de la Ley de Ohm para casos límites de la resistencia de un conductor, que lleva a los estudiantes a ignorar lo que ocurre en el circuito cuando la resistencia intermedia se anula y se convierte en un cable de conexión, o cuando la resistencia tiende a infinito y actúa como un interruptor abierto.

A continuación se muestran algunos ejemplos de concepciones alternativas o razonamientos acientíficos (Categoría II / MA) expresados por los estudiantes en tales ítems: (Ej.1.1) "La bombilla B1 brilla más que B2 porque se produce gasto de corriente en la resistencia R"; (Ej. 1.2) "Supongo que L1 brillará más que L2 y que ambas aumentarán su brillo si no hay resistencia"; (Ej. 1.3) "Creo que ahora disminuye el brillo de la bombilla B1, pero B2 dejará de brillar".

\subsection{Análisis de un circuito de varios elementos en paralelo}

En segundo lugar se analizan las respuestas de los alumnos a las tres preguntas planteadas en el problema P2, mostrado en la parte superior de la Tabla 2, en el que se plantea el análisis de un circuito de varios elementos conectados en paralelo, formado por un generador ideal, dos lámparas iguales y una resistencia $\mathrm{R}$, que puede permanecer constante o cambiar su valor. En la parte inferior de la tabla se muestran los resultados del análisis de frecuencias (y porcentajes) derivados de la categorización de ideas en cada uno de tales ítems, con arreglo a las mismas categorías consideradas anteriormente (I, II, III y IV).

Tabla 2. Resultados del análisis de un circuito de varios elementos en paralelo

\footnotetext{
2. El circuito de la figura está formado por un generador ideal G (que proporciona una tensión constante $\mathrm{V}_{\mathrm{o}}$ ) y dos lámparas iguales L1 y L2 (con una resistencia interna de valor $\mathrm{R}_{\mathrm{L}}$ ) en paralelo con una resistencia externa variable, cuyo valor inicial es igual que la resistencia de las lámparas $\left(\mathrm{Rv}=\mathrm{R}_{\mathrm{L}}\right)$

2.1. Explicar cuál de tales lámparas brilla más al principio.

2.2. Predecir si se produce algún cambio en el brillo de ambas lámparas cuando el valor de la resistencia externa aumenta hasta el doble de su valor inicial $\left(R v=2 R_{L}\right)$.

2.3. Predecir qué ocurre en el circuito cuando el valor de la resistencia externa disminuye hasta cero (equivalente a un cable sin resistencia).
}

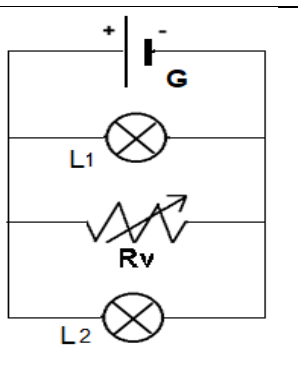

\begin{tabular}{|c|c|c|c|c|c|c|c|c|}
\hline \multicolumn{9}{|c|}{ Frecuencias y Porcentajes por Categorías de Respuesta } \\
\hline Ítems & \multicolumn{2}{|c|}{ I (MI) } & \multicolumn{2}{|c|}{ II (MA) } & \multicolumn{2}{|c|}{ III (MCi) } & \multicolumn{2}{|c|}{ IV $(\mathrm{MCa})$} \\
\hline 2.1 & 8 & 9,9 & 24 & 29,6 & 26 & 32,1 & 23 & 28,4 \\
\hline 2.2 & 12 & 14,8 & 35 & 43,4 & 21 & 25,9 & 14 & 17,3 \\
\hline 2.3 & 15 & 18,5 & 42 & 51,8 & 15 & 18,5 & 9 & 11,1 \\
\hline
\end{tabular}

MI = Mod. indefinido; MA = Mod. alternativo $\quad \mathrm{MCi}=$ Mod. Científico incompleto; $\mathrm{MCa}=$ Mod. científico aceptable

Cuando los estudiantes han de predecir cuál de las dos lámparas brilla más en dicho montaje (ítem 2.1) se aprecia la existencia de razonamientos alternativos e ideas erróneas desde el punto de vista científico (algo menos de un tercio), junto con algunas respuestas en blanco o explicaciones confusas (alrededor de una décima parte). Respecto a los cambios que se producen en el brillo de las lámparas al aumentar el valor de la resistencia intermedia (ítem 2.2) los resultados son más bajos que en el ítem anterior pues afecta a más de $2 / 5$ de la muestra y todavía son más bajos en la pregunta sobre los cambios que se producen cuando la resistencia intermedia alcanza valores extremos hacia cero o hacia infinito (ítem 2.3), ya que el porcentaje de concepciones alternativas supera a la mitad de las respuestas. 
Los principales modelos mentales y explicaciones de carácter alternativo que se aprecian en estas preguntas se refieren en primer lugar al llamado efecto topológico, combinado con el ya conocido modelo de consumo de la corriente, por el que muchos estudiantes creen que la corriente se va debilitando y llega menos intensidad a los elementos que se encuentran más alejados del generador. En segundo lugar apunta al desconocimiento de las leyes de asociación de elementos en paralelo (que están todos sometidos al mismo voltaje o diferencia de potencial) junto a razonamientos de tipo local y secuencial que lleva a los estudiantes a considerar solo cambios en las magnitudes físicas de los elementos donde se produce una modificación y se ignora lo que ocurre en el resto del circuito. En en tercer lugar, estos resultados se pueden deber a la ya citada falta de comprensión de la Ley de Ohm para casos límites de la resistencia de un conductor, lo cual les lleva a muchos a ignorar que se produce un cortocircuito cuando la resistencia intermedia se anula, o que se convierte en un circuito de dos lámparas cuando la resistencia tiende a infinito y actúa como un interruptor abierto.

A continuación se muestran algunos ejemplos de concepciones alternativas o razonamientos acientíficos (Categoría II / MA) expresados por los estudiantes en tales ítems: (Ej.2.1) "La bombilla L1 brilla más porque está situada delante de la resistencia y a B2 llega la corriente más débil".; (Ej. 2.2) "Ambas lámparas reducen su iluminación, pero B2 brilla menos que B1 por estar detrás"; (Ej. 2.3) "Creo que la bombilla L1 brilla igual que antes y que L2 deja de brillar porque ahora la resistencia no deja pasar corriente.".

\subsection{Análisis de un circuito mixto}

En tercer lugar se analizan las respuestas de los alumnos a las cuatro cuestiones planteadas en el problema P3, que se muestra en la parte superior de la Tabla 3. Ahora se trata de analizar un circuito formado por un generador ideal, dos lámparas iguales y una resistencia $\mathrm{R}$ constante o variable, que están conectados en un montaje mixto. En la parte inferior de la tabla se muestran los resultados del análisis de frecuencias (y porcentajes) derivados de la categorización de ideas en cada una de estas cuestiones, considerando las cuatro categorías de respuestas previstas en todo el estudio.

Tabla 3. Resultados del análisis de un circuito de varios elementos en montaje mixto

3. En la figura se muestra un circuito mixto formado por un generador ideal $\mathrm{G}$ que proporciona una tensión constante Vo, dos lámparas iguales L1 y L2 que tienen la misma resistencia $R_{L}$, y una resistencia variable $R v$, cuyo valor inicial es igual que la resistencia de las lámparas $\left(\mathrm{Rv}=\mathrm{R}_{\mathrm{L}}\right)$.

3.1. Explicar si la intensidad de corriente eléctrica que circula por la lámpara L1 es igual o diferente que en la lámpara L2.

3.2. Si en un determinado instante aumenta el valor de Rv hasta el doble de su valor inicial, explicar si se produce algún cambio en la intensidad de corriente eléctrica

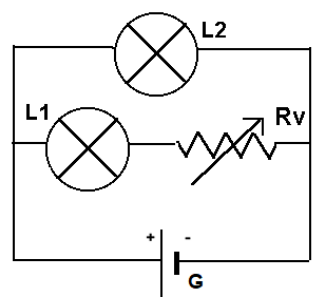
que suministra el generador al circuito.

3.3. Explicar si se produce algún cambio en el brillo o la potencia de la lámpara L2, al aumentar el valor de Rv.

3.4. Explicar si se produce algún cambio en la diferencia de potencial de la resistencia Rv y en el brillo de la lámpara L1, al aumentar el valor de Rv.

\begin{tabular}{|c|c|c|c|c|c|c|c|c|}
\hline \multicolumn{9}{|c|}{ Frecuencias y Porcentajes por Categorías de Respuesta } \\
\hline \multirow{2}{*}{$\begin{array}{c}\text { Ítems } \\
3.1\end{array}$} & \multicolumn{2}{|c|}{ I (MI) } & \multicolumn{2}{|c|}{ II (MA) } & \multicolumn{2}{|c|}{ III (MCi) } & \multicolumn{2}{|c|}{ IV (MCa) } \\
\hline & 15 & 18,5 & 41 & 50,6 & 16 & 19,8 & 10 & 12,3 \\
\hline 3.2 & 12 & 14,8 & 58 & 71,6 & 7 & 8,6 & 4 & 4.9 \\
\hline 3.3 & 14 & 17,3 & 50 & 61,7 & 6 & 7,4 & 11 & 13,6 \\
\hline 3.4 & 13 & 16,1 & 56 & 69,1 & 9 & 11,1 & 3 & 3,7 \\
\hline
\end{tabular}

$\mathrm{MI}=$ Mod. indefinido; $\mathrm{MA}=$ Mod. alternativo; $\mathrm{MCi}=$ Mod. Científico incompleto; $\mathrm{MCa}=$ Mod. científico aceptable 
Con relación a la intensidad de corriente que circula por las dos lámparas del circuito tras la bifurcación del primer nudo (ítem 3.1), se aprecia un nivel bastante elevado de predicciones erróneas desde el punto de vista científico y de modelos mentales de carácter alternativo (más de la mitad), junto con bastantes respuestas en blanco y explicaciones confusas (una sexta parte). Respecto a los cambios que se producen en la intensidad de corriente del generador al aumentar el valor de la resistencia variable (ítem 3.2) los resultados son los más bajos de todo el cuestionario, ya que los modelos mentales alternativos afectan a más del setenta por ciento de las respuestas. Algo parecido ocurre al hacer predicciones sobre los cambios producidos en el brillo de la primera lámpara (ítem 3.3), donde las concepciones alternativas afectan a más tres quintas partes, o al predecir los cambios que ocurren en el brillo de la segunda lámpara y en el voltaje de la resistencia cuando ésta aumenta (ítem 3.4), ya que de nuevo los razonamientos de carácter alternativo suponen algo más de dos tercios de las respuestas.

En los modelos de carácter alternativo que se aprecian en estas preguntas se combinan una serie de aspectos que se relacionan de nuevo con el modelo de consumo de la corriente eléctrica, con el razonamiento de tipo local y secuencial, o con la falta de comprensión significativa de las leyes de Kirchoff (sobre todo la segunda ley). También se aprecia en estos resultados la notable confusión entre las magnitudes intensidad de corriente y voltaje o diferencia de potencial, que influye considerablemente en la idea de que un generador ideal de tensión proporciona una intensidad de corriente al circuito, independientemente de los cambios que se produzcan en su estructura o en el valor de alguna de sus variables independientes.

A continuación se muestran algunos ejemplos de concepciones alternativas o razonamientos acientíficos (Categoría II /MA) expresados por los estudiantes en tales ítems: (Ej.3.1) "En toda bifurcación se divide la corriente en dos partes iguales"; "la intensidad de corriente es igual en las dos lámparas pero después de L1 se reduce al pasar por la resistencia". (Ej. 3.2) "Al crecer el valor de Rv el generador tiene que aportar una corriente mayor"; "A medida que aumenta la resistencia hay más gasto de energía y se necesita una corriente más fuerte". (Ej. 3.3) "A medida que aumenta la resistencia es mayor el gasto de energía y las lámparas van brillando menos"; "al crecer la resistencia la corriente que se reparte es menor". (Ej.3.4) "Cuando aumenta la resistencia disminuye el valor de la corriente en esa rama y disminuyen tanto el brillo de la lámpara como el voltaje de Rv".

\subsection{Modelos mentales relacionados con el análisis de relaciones macro-micro en un circuito simple}

En la Tabla 4 se muestra el enunciado y el montaje del cuarto problema (P4), correspondiente a un circuito formado por un generador ideal de tensión, un par de cables de conexión (de resistencia despreciable) y una barra metálica de resistencia R. También se muestran los datos derivados del análisis de frecuencias en los items del P4 correspondiente a los 4 tipos de respuesta que se han indicado antes.

Con relación a si existe diferencia de potencial eléctrico en los cables A y B o en la barra metálica (ítem 4.1) se aprecia un alto porcentaje de concepciones alternativas o respuestas erróneas desde el punto de vista científico (cerca de la mitad) y bastantes respuestas en blanco o explicaciones confusas (casi una sexta parte). Respecto a si existe campo eléctrico en los cables A y B o en la barra metálica (ítem 4.2) los resultados son aun más bajos y algo similar ocurre en la pregunta sobre la densidad de corriente en los cables A y B o en la barra metálica (ítem 4.3).

Las principales dificultades y concepciones alternativas apreciadas en estas preguntas se refieren a la confusión entre corriente eléctrica y voltaje o diferencia de potencial, la incomprensión del papel que desempeña el campo eléctrico en el transporte de carga eléctrica en los circuitos y su relación con la diferencia de potencial entre dos puntos, o la confusión entre densidad e intensidad de corriente, la ausencia de relación entre el campo eléctrico y la densidad de corriente a nivel microscópico y, sobre 
todo, la idea de que hay consumo de corriente (tanto en intensidad como en densidad) al pasar por la barra de metal. Estos hechos indican que los estudiantes apenas poseen conocimientos previos adecuados sobre el modelo científico de corriente eléctrica a nivel avanzado, ignorando las importantes relaciones entre magnitudes eléctricas de carácter macroscópico y microscópico.

Tabla 4. Resultados del análisis de relaciones macro-micro en un circuito básico

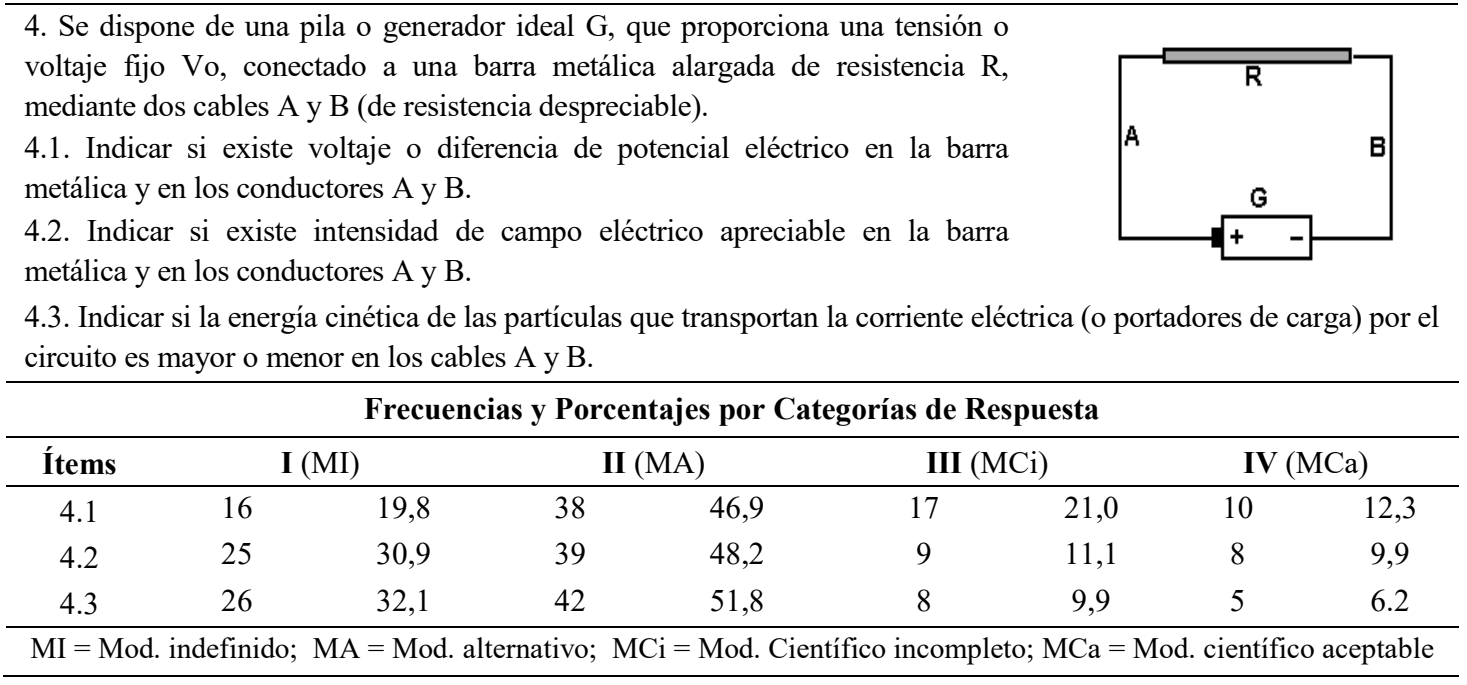

A continuación se muestran algunos ejemplos de concepciones alternativas o razonamientos acientíficos (Categoría II /MA) expresados por los estudiantes en tales ítems: (Ej.4.1) " El voltaje llega a todo los elementos del circuito, pero hay menos gasto de voltios en los cables A y B que en la barra metálica"; (Ej. 4.2) "Hay campo eléctrico entre el polo positivo y el negativo del generador y en el resto del circuito sólo hay corriente y voltaje"; (Ej. 4.3) " Las cargas eléctricas de la corriente pierden parte de su fuerza al atravesar la resistencia".

\subsection{Modelos mentales relacionados con el análisis de un circuito $\mathrm{RC}$-Serie en régimen transitorio}

Finalmente se analizan las respuestas de los alumnos a los tres ítems del problema P5, mostrado en la Tabla 5. Aquí se trata de analizar un circuito formado por un generador ideal de tensión conectado en serie con una resistencia $\mathrm{R}$, un condensador $\mathrm{C}$ y un interruptor. Cuando se cierra el interruptor los estudiantes deben hacer predicciones sobre el comportamiento de diversas magnitudes del circuito. En la tabla también se muestran los resultados del análisis de frecuencias (y porcentajes) derivados de la categorización de ideas mostradas por los estudiantes en tales cuestiones, manteniendo las mismas categorías de respuestas (I, II, III y IV) de los problemas anteriores.

Con respecto a la variación de la intensidad de corriente durante el proceso de carga de un circuito RCserie (ítem 5.1) también se aprecia un alto porcentaje de modelos alternativos o ideas erróneas desde el punto de vista científico (cerca de tres quintos en la categoría II) y bastantes respuestas en blanco o explicaciones confusas (más de una quinta parte en I), resultando muy bajo el nivel de respuestas adecuadas (IV) o al menos aproximadas (III), pues éstas en conjunto suponen solo un décimo del conjunto de respuestas analizadas. Unos resultados parecidos, pero un poco más bajos, se obtienen en las predicciones sobre el voltaje o diferencia de potencial eléctrico existente en las placas del condensador durante el proceso de carga (ítem 5.2), aunque el número de respuestas correctas o aceptables se eleva un poco hasta alcanzar cerca de una sexta parte en las predicciones sobre la descarga de un condensador a través de un cable sin resistencia y la reestructuración final del circuito (ítem 5.3). 
Tabla 5. Resultados del análisis de un circuito $R C$-Serie en régimen transitorio

5. El montaje de la figura está formado por un generador ideal G (que proporciona una tensión fija $\mathrm{Vo}$ ), conectado en serie con un condensador de gran capacidad $\mathrm{C}$ y una lámpara de gran resistencia $\mathrm{R}$, se cierra el interruptor en el instante $\mathrm{t}=0$.

5.1. Indicar qué ocurre con la intensidad de corriente que circula por la resistencia tras cerrar el interruptor.

5.2. Indicar qué ocurre con la diferencia de potencial o voltaje en las placas del condensador tras cerrar el interruptor.

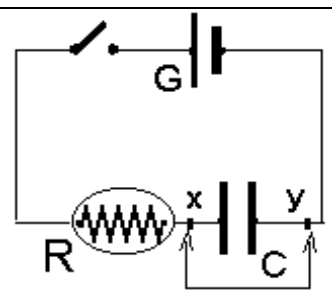

5.3. Cuando ha pasado un tiempo suficientemente largo, se conecta un cable (sin resistencia) entre los puntos X e Y. Indicar si experimenta algún cambio el voltaje del condensador y el brillo de la lámpara.

\begin{tabular}{|c|c|c|c|c|c|c|c|c|}
\hline \multicolumn{9}{|c|}{ Frecuencias y Porcentajes por Categorías de Respuesta } \\
\hline Ítems & \multicolumn{2}{|c|}{ I (MI) } & \multicolumn{2}{|c|}{ II (MA) } & \multicolumn{2}{|c|}{ III (MCi) } & \multicolumn{2}{|c|}{ IV $(\mathrm{MCa})$} \\
\hline 5.1 & 22 & 27,2 & 50 & 61,7 & 6 & 7,4 & 3 & 3,7 \\
\hline 5.2 & 21 & 25,9 & 48 & 59,3 & 8 & 9,9 & 4 & 4,9 \\
\hline 5.3 & 19 & 23,5 & 46 & 56,8 & 10 & 12,3 & 6 & 7,4 \\
\hline
\end{tabular}

Las principales dificultades de aprendizaje e ideas de carácter alternativo que se aprecian en estas preguntas se asocian con la idea de que la intensidad de corriente no cambia en el circuito (porque la confunden con el voltaje del generador que es constante), que el voltaje del condensador es constante por estar conectado al generador que proporciona una tensión fija, o que al conectar un cable a los extremos del condensador no se ve afectado el resto del circuito. Junto a estos modelos de pensamiento de carácter alternativo también se aprecian otros razonamientos erróneos de menor extensión y muchas ideas confusas o incoherentes. Estos resultados muestran que los estudiantes poseen escasos conocimientos previos adecuados sobre los procesos de carga y descarga de condensadores en circuitos de corriente continua o, en general, sobre los circuitos eléctricos en régimen transitorio que tienen mayor relación con el modelo científico de corriente eléctrica a nivel avanzado o microscópico (Pontes, 2017).

A continuación se muestran algunos ejemplos de concepciones alternativas o razonamientos acientíficos (Categoría II /MA) expresados por los estudiantes en tales ítems: (Ej.5.1) "El generador de corriente continua produce una corriente fija y el circuito consume siempre la misma energía"; (Ej. 5.2) "El voltaje de la fuente es fijo y se reparte entre la resistencia y el condensador desde el principio"; (Ej. 5.3) "La corriente de la lámpara no cambia pero no puede pasar por el condensador y circula por el cable que no tiene resistencia ni voltaje".

\section{Conclusiones}

En este trabajo se han mostrado los primeros resultados de una investigación destinada a evaluar los conocimientos previos de los estudiantes de $1^{\circ}$ curso de ingeniería en el tema de electrocinética y a analizar los modelos de razonamiento que utilizan los alumnos al abordar problemas cualitativos sobre el funcionamiento de diferentes tipos de circuitos eléctricos. Para ello se ha utilizado un cuestionario de preguntas abiertas, que los estudiantes han cumplimentado a través de la plataforma Moodle, antes de recibir instrucción formal sobre el tema.

Los resultados obtenidos en este estudio indican que los alumnos utilizan modelos de pensamiento superficial y expresan concepciones alternativas que ponen de manifiesto la falta de comprensión del modelo científico de corriente eléctrica, en los estudios previos a la enseñanza universitaria. Estos hechos 
tienen implicaciones notables para la enseñanza de la electricidad, que es un tema de la física de gran importancia para la formación de ingenieros, mostrando la necesidad de desarrollar propuestas metodológicas que contribuyan a mejorar la calidad de la educación científica, tratando de favorecer el cambio conceptual y la progresión de los modelos de pensamiento de los alumnos en esta temática (Balta, 2015; Pontes, 2017; Yuliati, Riantoni y Mufti, 2018).

Esta perspectiva educativa supone llevar a cabo una serie de innovaciones didácticas importantes, entre las que podemos destacar las siguientes: a) diagnosticar las concepciones alternativas que utilizan los alumnos en el aprendizaje de la electricidad e identificar aquellas que más se resisten a ser cambiadas; b) dedicar mayor atención durante el proceso de instrucción a promover situaciones de aprendizaje que cuestionen los modelos mentales alternativos que se han detectado en este estudio; c) desarrollar estrategias y recursos didácticos destinados a favorecer la progression de ideas de los alumnos, trasformando sus modelos de pensamiento inicial en modelos científicos; d) evaluar si al aplicar tales recursos se llega a producir la evolución conceptual deseada e introducir las modificaciones oportunas para que las propuestas metodológicas sean cada vez más adecuadas y útiles.

Como consecuencia de tales planteamientos, en la siguiente etapa del proyecto de investigación estamos ensayando una propuesta metodológica orientada a mejorar el proceso de aprendizaje de modelos físicos, utilizando nuevos materiales didácticos y recursos TIC interactivos como Moodle, CmapTools y Laboratorios Virtuales que han mostrado bastante utilidad para favorecer el aprendizaje de conceptos y destrezas en otros temas de Física (Yuliati et al., 2018;.Pontes, 2020). En trabajos posteriores se mostrarán los resultados obtenidos en la aplicación de tales innovaciones.

Agradecimientos: Este estudio forma parte de una investigación de varios años que ha contado con la ayuda asignada a un Proyecto de Innovación Educativa (UCO-2017-1-5010) y un Proyecto de I+D+i del Ministerio: EDU2017-82518-P (Convocatoria de 2018-2021)

\section{Referencias Bibliográficas}

ALDUCIN, J.M. y VÁZQUEZ, A.I. (2014). "Mejora del rendimiento en Ingeniería a través de blended-learning". Digital Education Review, 25, 87-107.

BALTA, N. (2015). "Development of 3-D Mechanical Models of Electric Circuits and Their Effect on Students' Understanding of Electric Potential Difference". European Journal of Physics Education, 6(19), 15-24

CLEMENT, J.J. (2000). "Model based learning as a key research area for science education". International Journal of Science Education, 22(9), 1041-1053.

CUDMANI, L.C. y FONTDEVILLA, P.A. (1990). "Concepciones previas en el aprendizaje significativo del electromagnetismo”. Enseñanza de las Ciencias, 8 (3), 215-222

DUIT, R., JUNG, W. y RHÖNECK, C. (1985). Aspects of Understanding Electricity. Kiel: Institute for Science Education.

GUNSTONE, R., MULHALL, P. y MCKITTRICK, B. (2009). "Physics Teachers' Perceptions of the Difficulty of Teaching Electricity”. Research in Science Education, 39(4), 515-538

HIERREZUELO, J. y MONTERO, A. (1990). La ciencia de los alumnos. Velez-Málaga: Elzevir. 
LÓPEZ-DONOSO, E. y SILVA, R. (2015). "Transformación de los modelos mentales sobre los conceptos de fuerza y campo eléctrico mediante la metodología Webquest, en estudiantes universitarios de Ingeniería”. Caderno Brasileiro de Ensino de Física, 32 (1), 2-31

LÓPEZ-QUINTERO, J.L., VARO, M. y PONTES, A. (2018). "La exploración de conocimientos previos de los estudiantes en la enseñanza científico-técnica universitaria mediante recursos TIC interactivos". En López-García, C. y Manso, J. (Eds.) Transforming education for a changing world (pp. 96-105); Eindhoven: Adaya Press.

MEI-HUNG, CH. y JING-WEN, L. (2005). "Promoting Fourth Graders' Conceptual Change of Their Understanding of Electric Current via Multiple Analogies”. Journal of Research in Science Teaching, 42(4), 429-464.

METIOUI, A., BRASSARD, C., LEVASSEUR, J. y LAVOIE, M. (1996). “The persistence of students' unfunded beliefs about electrical circuits: the case of Ohm's law”. International Journal of Science Education, 18 (2), $193-212$.

OLIVA, J.M. (2019). "Distintas acepciones para la idea de modelización en la enseñanza de las ciencias. Enseñanza

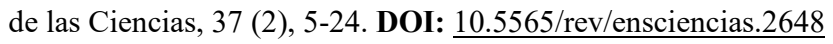

PONTES, A. y DE PRO, A. (2001). “Concepciones y razonamientos de expertos y aprendices sobre electrocinética: consecuencias para la enseñanza y la formación de profesores”. Enseñanza de las Ciencias, 19 (1), 103-122.

PONTES, A. (2014). "Representación del conocimiento físico del alumnado universitario mediante mapas conceptuales elaborados con CmapTools". Alambique: Didáctica de las Ciencias Experimentales. 76, 34-42.

PONTES, A. (2017). "El uso de simulaciones interactivas para comprender el modelo de corriente eléctrica". Enseñanza de las Ciencias, 35( $\mathrm{N}^{\circ}$ Extra), 4371-4377.

PONTES, A. (2020). "Recursos TIC para la innovación educativa en la enseñanza de la física universitaria". III International Seminar of Science Education (en prensa). Oporto: APEduC

PONTES, A., VARO, M., BLANCA, A., PEDRÓS, G., GARCÍA, M.C. y AGUILERA, M.J. (2019). Modelos físicos en electricidad y actividades de aprendizaje. Córdoba: Ediciones Don Folio.

TREAGUST, D.F., CHITTLEBOROUGH, G. y MAMIALA, T.L. (2002). "Students' understanding of the role of scientific models in learning science”. International Journal of Science Education, 24(4), 357-368.

YULIATI, L., RIANTONI, C. y MUFTI, N. (2018). "Problem Solving Skills on Direct Current Electricity through Inquiry-Based Learning with PhET Simulations". International Journal of Instruction, 11(4), 123-138.

ZEYNEP, U. y IBILGE, D. (2011). "The Effect of Combining Analogy-Based Simulation and Laboratory Activities on Turkish Elementary School Students' Understanding of Simple Electric Circuits". Turkish Online Journal of Educational Technology, 10 (4), 320-329. 